\title{
A type of the travelling wave solutions of higher-order Camassa-Holm equations
}

\author{
Fenggang Xue ${ }^{1}$ Danping Ding ${ }^{1}$ \\ ${ }^{1}$ College of Sciences, Jiangsu University, Zhenjiang 212013, China.
}

\begin{abstract}
This paper researches the wave equation of higher-order Camassa-Holm equations, the general expression of a type of travelling wave solutions is obtained.
\end{abstract}

Keywords: higher-order Camassa-Holm equations, the wave equation, the travelling wave solutions

\section{Introduction}

In 2003,Adrian Constantin and Boris Kolev first got the higher-order Camassa-Holm equations ${ }^{[1]}$. Its concrete form is as follows:

$$
\partial_{t} u=B_{k}(u, u)
$$

where $k \in\{0\} \cup N$,

$$
\begin{gathered}
B_{k}(u, u):=A_{k}{ }^{-1} C_{k}(u)-u \partial_{x} u, \\
A_{k}(u):=\sum_{j=0}^{k}(-1)^{j} \partial_{x}{ }^{2 j} u, \\
C_{k}(u):=-u A_{k}\left(\partial_{x} u\right)+A_{k}\left(u \partial_{x} u\right)-2 \partial_{x} u A_{k}(u)
\end{gathered}
$$

In 2009, G.M.Coclite,H.Holden and K.H.Karlsen first researched the global Well-Posedness of the higher-order Camassa-Holm equations, and Got a weak solution $^{[2]}$.

In 2010, Dingdanping, Lvpeng researched the existence of global solutions to the higher-order Camassa-Holm equations. Global solution is constructed by the small viscosity method for the frequency localized equations, especially global solution is energy conservative for given finite band initial data ${ }^{[3]}$.

The structure of the paper is organized as follows. In Section 2, the formal solutions of higher-order $\mathrm{C}-\mathrm{H}$ equations are obtained, In Section 3,Energy Conservation of the formal solutions is discussed. In Section 4, a type of travelling wave solutions are given.

\section{The formal solution}

According to the equation (1),we have

$$
\begin{aligned}
& \sum_{j=0}^{k}(-1)^{j}\left[\partial_{x}{ }^{2 j}\left(\partial_{t} u\right)+u \partial_{x}{ }^{2 j}\left(\partial_{x} u\right)+2 \partial_{x} u \partial_{x}{ }^{2 j} u\right] \\
& =0
\end{aligned}
$$

Let $u=\varphi(\xi)=\varphi(x-c t)$, the travelling wave equation of (2) is

$$
\sum_{j=0}^{k}(-1)^{j}\left[\left(-c \varphi^{\prime}\right)^{(2 j)}+\varphi\left(\varphi^{\prime}\right)^{(2 j)}+2 \varphi^{\prime} \varphi^{(2 j)}\right]=0
$$

After twice-integration of equation (3),it was differentiated to yield

$$
\begin{aligned}
\varphi^{\prime}\left(\varphi^{(2 k)}-\right. & \varphi^{(2 k-2)}+\varphi^{(2 k-4)}-\cdots \\
\left.-(-1)^{k} \varphi^{\prime \prime}+(-1)^{k} \varphi\right) & =0,
\end{aligned}
$$

if $\varphi^{\prime}=0$, then $u=a$ ( $a$ is an arbitrary real number), otherwise, 
$\varphi^{(2 k)}-\varphi^{(2 k-2)}+\cdots-(-1)^{k} \varphi^{\prime \prime}+(-1)^{k} \varphi=0$.

Equation (4) is ODE, when $k=0$,the solution is $u=a$ ( $a$ is an arbitrary real number).For $k \geq 1$, its characteristic equation is

$$
\begin{gathered}
r^{2 k}-r^{2 k-2}+r^{2 k-4}-\cdots-(-1)^{k} r^{2} \\
+(-1)^{k}=0 .
\end{gathered}
$$

According to references [4-6], the roots of (5) have the form as follows:

$$
r_{i}= \pm \alpha_{i} \pm i \beta_{i}
$$

where $\left\|r_{i}\right\|=\sqrt{\alpha_{i}^{2}+\beta_{i}^{2}}=1, a_{i}>0, \beta_{i}>0$,a ndwhen $k$ is even, $i=1,2, \cdots \frac{k}{2}$, when $k$ is odd, $i=1,2, \cdots \frac{k+1}{2}$.

So when $k$ is even,the formal solution of (2) is

$$
\begin{aligned}
& u(x, t)=\sum_{i=1}^{k / 2}\left[e ^ { \alpha _ { i } ( x - c t ) } \left(c_{i 1} \cos \beta_{i}(x-c t)+\right.\right. \\
& \left.\quad c_{i 2} \sin \beta_{i}(x-c t)\right)+e^{-\alpha_{i}(x-c t)}\left(c_{i 3} \cos \beta_{i}(x-c t)\right. \\
& \left.\left.\quad+c_{i 4} \sin \beta_{i}(x-c t)\right)\right]
\end{aligned}
$$

where $c_{i 1}, c_{i 2}, c_{i 3}, c_{i 4}$ are coefficients,

when $k$ is odd,the formal solution of (2) is

$$
\begin{aligned}
& u(x, t)=\sum_{i=1}^{(k+1) / 2}\left[e ^ { \alpha _ { i } ( x - c t ) } \left(c_{i 1} \cos \beta_{i}(x-c t)+\right.\right. \\
& \left.\quad c_{i 2} \sin \beta_{i}(x-c t)\right)+e^{-\alpha_{i}(x-c t)}\left(c_{i 3} \cos \beta_{i}(x-c t)\right. \\
& \left.\left.\quad+c_{i 4} \sin \beta_{i}(x-c t)\right)\right]
\end{aligned}
$$

where $c_{i 1}, c_{i 2}, c_{i 3}, c_{i 4}$ are coefficients.

\section{Energy Conservation}

According to attenuation of the travelling wave solutions, the formal solution can be reduced to

$$
\begin{aligned}
u(x, t)= & \sum_{i=1}^{k / 2} e^{-\left|\alpha_{i}(x-c t)\right|}\left[c_{i 1} \cos \beta_{i}(x-c t)\right. \\
& \left.+c_{i 2} \sin \beta_{i}(x-c t)\right](k \text { is even }), \\
u(x, t)= & \sum_{i=1}^{(k+1) / 2} e^{-\left|\alpha_{i}(x-c t)\right|}\left[c_{i 1} \cos \beta_{i}(x-c t)\right. \\
& \left.+c_{i 2} \sin \beta_{i}(x-c t)\right](k \text { is odd })
\end{aligned}
$$

if $u(x, t)$ obeys conservation of energy while $k$ is even,then when $k$ is odd, the same conclusion can be obtained.

Let $I(t)=\int_{R} u^{2}+\left(\partial_{x} u\right)^{2}+\cdots+\left(\partial_{x}^{k} u\right)^{2} d x$,

$$
\text { then } \begin{aligned}
\frac{d I(t)}{d t} & =\sum_{l=0}^{k} \int_{R} \partial_{x}^{l} u \partial_{x}^{l}\left(\partial_{t} u\right) d x\left(\partial_{t} u=-c \partial_{x} u\right) \\
& =-c \cdot \sum_{l=0}^{k} \int_{R} \partial_{x}^{l} u \partial_{x}^{l+1} u d x
\end{aligned}
$$

if $u(x, t)$ satisfies the condition of $\frac{d I(t)}{d t}=0$ in a certain assumptions, a type of travelling wave solutions can be obtained. So two independent assumed conditions are given.

3.1. $c_{i 2}=\mathbf{0}$

when $k$ is even $(k \neq 0)$,then

$$
\begin{aligned}
& \int_{-\infty}^{c t} \partial_{x}^{l} u \partial_{x}^{l+1} u d x \\
& \quad=\sum_{1 \leq i, j \leq \frac{k}{2}} c_{i 1} c_{j 1} \int_{-\infty}^{c t} e^{\left(\alpha_{i}+\alpha_{j}\right)(x-c t)} \cos \left(l \theta_{i 1}+\beta_{i}(x-c t)\right)
\end{aligned}
$$

$$
\cos \left(\theta_{j 1}+l \theta_{j 1}+\beta_{j}(x-c t)\right) d x,
$$


where $\cos \theta_{i 1}=a_{i}, \sin \theta_{i 1}=\beta_{i}, l=0,1,2, \cdots, k$,

$$
\begin{aligned}
\int_{-\infty}^{c t} e^{\left(\alpha_{i}+\alpha_{j}\right)(x-c t)} \cos \left(l \theta_{i 1}+\beta_{i}(x-c t)\right) \cdot & \cos \left(\theta_{j 1}+l \theta_{j 1}+\beta_{j}(x-c t)\right) d x \\
= & \frac{1}{2} \cos \left(l \theta_{i 1}+\theta_{j 1}+l \theta_{j 1}\right) \frac{\alpha_{i}+\alpha_{j}}{\left(\alpha_{i}+\alpha_{j}\right)^{2}+\left(\beta_{i}+\beta_{j}\right)^{2}} \\
+ & \frac{1}{2} \sin \left(l \theta_{i 1}+\theta_{j 1}+l \theta_{j 1}\right) \frac{\left(\beta_{i}+\beta_{j}\right)}{\left(\alpha_{i}+\alpha_{j}\right)^{2}+\left(\beta_{i}+\beta_{j}\right)^{2}} \\
+ & \frac{1}{2} \cos \left(l \theta_{i 1}-\theta_{j 1}-l \theta_{j 1}\right) \frac{\alpha_{i}+\alpha_{j}}{\left(\alpha_{i}+\alpha_{j}\right)^{2}+\left(\beta_{i}-\beta_{j}\right)^{2}} \\
+ & \frac{1}{2} \sin \left(l \theta_{i 1}-\theta_{j 1}-l \theta_{j 1}\right) \frac{\left(\beta_{i}-\beta_{j}\right)}{\left(\alpha_{i}+\alpha_{j}\right)^{2}+\left(\beta_{i}-\beta_{j}\right)^{2}}
\end{aligned}
$$$$
\begin{aligned}
& \int_{c t}^{+\infty} \partial_{x}^{l} u \partial_{x}^{l+1} u d x \\
& \quad=\sum_{1 \leq i, j \leq \frac{k}{2}} c_{i 1} c_{j 1} \int_{c t}^{+\infty} e^{-\left(\alpha_{i}+\alpha_{j}\right)(x-c t)} \cos \left(l \theta_{i 2}+\beta_{i}(x-c t)\right) .
\end{aligned}
$$$$
\cos \left(\theta_{j 2}+l \theta_{j 2}+\beta_{j}(x-c t)\right) d x,
$$$$
\text { where } \cos \theta_{i 2}=-a_{i}, \sin \theta_{i 2}=\beta_{i}, \theta_{i 2}=\pi-\theta_{i 1} \text {. }
$$$$
\begin{gathered}
\int_{c t}^{+\infty} e^{-\left(\alpha_{i}+\alpha_{j}\right)(x-c t)} \cos \left(l \theta_{i 2}+\beta_{i}(x-c t)\right) \cdot \\
\cos \left(\theta_{j 2}+l \theta_{j 2}+\beta_{j}(x-c t)\right) d x \\
=-\frac{1}{2} \cos \left(l \theta_{i 1}+\theta_{j 1}+l \theta_{j 1}\right) \frac{\alpha_{i}+\alpha_{j}}{\left(\alpha_{i}+\alpha_{j}\right)^{2}+\left(\beta_{i}+\beta_{j}\right)^{2}}
\end{gathered}
$$$$
-\frac{1}{2} \sin \left(l \theta_{i 1}+\theta_{j 1}+l \theta_{j 1}\right) \frac{\left(\beta_{i}+\beta_{j}\right)}{\left(\alpha_{i}+\alpha_{j}\right)^{2}+\left(\beta_{i}+\beta_{j}\right)^{2}}
$$$$
-\frac{1}{2} \cos \left(l \theta_{i 1}-\theta_{j 1}-l \theta_{j 1}\right) \frac{\alpha_{i}+\alpha_{j}}{\left(\alpha_{i}+\alpha_{j}\right)^{2}+\left(\beta_{i}-\beta_{j}\right)^{2}}
$$

$$
-\frac{1}{2} \sin \left(l \theta_{i 1}-\theta_{j 1}-l \theta_{j 1}\right) \frac{\left(\beta_{i}-\beta_{j}\right)}{\left(\alpha_{i}+\alpha_{j}\right)^{2}+\left(\beta_{i}-\beta_{j}\right)^{2}}
$$

then $\int_{R} \partial_{x}^{l} u \partial_{x}^{l+1} u d x$

$$
\begin{aligned}
& =\int_{-\infty}^{c t} \partial_{x}^{l} u \partial_{x}^{l+1} u d x+\int_{c t}^{+\infty} \partial_{x}^{l} u \partial_{x}^{l+1} u d x \\
& =\sum_{1 \leq i, j \leq \frac{k}{2}} c_{i 1} c_{j 1} \cdot 0 \\
& =0
\end{aligned}
$$

then $\frac{d I(t)}{d t}=-c \cdot \sum_{l=0}^{k} \int_{R} \partial_{x}^{l} u \partial_{x}^{l+1} u d x=0$.

So when $k$ is even, $u(x, t)$ obeys conservation of energy. the same conclusion is obtained while $k$ is odd.

\section{2. $c_{i 1}=\mathbf{0}$}

In this case, the same method is used to proof that the formal solution is also obeys conservation of energy.

\section{Conclusions}

A type of the travelling wave solutions of higher-order Camassa-Holm equations is as follows:

when $k$ is even,

$$
\begin{aligned}
& u(x, t)=\sum_{i=1}^{k / 2} c_{i} e^{-\left|\alpha_{i}(x-c t)\right|} \cos \beta_{i}(x-c t), \\
& \text { or } u(x, t)=\sum_{i=1}^{k / 2} c_{i} e^{-\left|\alpha_{i}(x-c t)\right|} \sin \beta_{i}(x-c t) ;
\end{aligned}
$$

when $k$ is odd,

$$
\begin{gathered}
u(x, t)=\sum_{i=1}^{(k+1) / 2} c_{i} e^{-\left|\alpha_{i}(x-c t)\right|} \cos \beta_{i}(x-c t), \\
\text { or } \\
u(x, t)=\sum_{i=1}^{(k+1) / 2} c_{i} e^{-\left|\alpha_{i}(x-c t)\right|} \sin \beta_{i}(x-c t),
\end{gathered}
$$


where $c_{i}$ is an arbitrary real number, $\alpha_{i}>0$ and $\beta_{i}>0$, them are determined by the following equation:

$r^{2 k}-r^{2 k-2}+r^{2 k-4}-\cdots-(-1)^{k} r^{2}+(-1)^{k}=0$.

The solution of this equation is $r_{i}= \pm \alpha_{i} \pm i \beta_{i}\left(\alpha_{i}^{2}+\beta_{i}^{2}=1\right)$, when $k$ is even, the subscript $i=1,2,3, \cdots, \frac{k}{2}$, when $k$ is odd, the subscript $i=1,2,3, \cdots, \frac{k+1}{2}$ and $r_{(k+1) / 2}= \pm \alpha_{(k+1) / 2} \pm \beta_{(k+1) / 2}= \pm 1$.

Moreover, when $k=1$, the travelling wave solution is $u(x, t)=c_{1} e^{-|x-c t|}\left(c_{1}\right.$ is an arbitrary real number).It is the soliton solution $^{[7]}$ of Camassa-Holm equation which is obtained by Camassa and Holm.

\section{References}

[1] A.Constantin,B.Kolev. “Geodesic flow on the diffeomorphism group of the circle". Comment.Math.Helv, pp. 787-804,2003.
[2] G.M.Coclite, H.Holden, K.H.Karlsen. "Well-posedness of higher-order Camassa-Holm equations".Journal of Differential Equations,pp.929-963, 2009.

[3] Ding Danping,Lv Peng. “Conservative solutions for higher-order CamassaHolm equations".J. Math. Phys,pp.51 $-66,2010$.

[4] Yang Zongpei. “A Criterion on real roots of a real coefficient monadic equation of even number order". Journal of Nanchang University, pp.43-49,1982.

[5] Han Yongdong. "Probing the Solution of reciprocal equation". Journal of Jilin Radio and television university, pp.24-25.2011.

[6] An min,Peng Yamian,etc. "Study on the Solution of certain equation of higher degree in mathematics". Science and Technology Information, pp.134+121, 2007.

[7] R.Camassa,D.D.Holm."An integrable shallow water equation with peaked solutions".Phys. Rev. Lett,pp.1661$1664,1993$. 